\title{
The impact of disease characteristics on multiple sclerosis patients' quality of life
}

\author{
Aziz Rezapour ${ }^{1,2}$, Abdollah Almasian Kia ${ }^{2}$, Sahar Goodarzi ${ }^{3,4}$, Mojtaba Hasoumi², \\ Soraya Nouraei Motlagh ${ }^{5}$, Sajad Vahedi ${ }^{6}$ \\ ${ }^{1}$ Health Management and Economics Research Center, Iran University of Medical Sciences, Tehran, Iran; ${ }^{2}$ Department of Health Economics, \\ School of Health Management and Information Sciences, Iran University of Medical Sciences, Tehran, Iran; ${ }^{3}$ Department of Health Economics, \\ School of Management and Information Sciences, Shiraz University of Medical Sciences, Shiraz, Iran; ${ }^{4}$ Student Research Committee, Shiraz \\ University of Medical Sciences, Shiraz, Iran; ${ }^{5}$ Public Health Department, School of Health and Nutrition, Social Determinants of Health Research \\ Center, Lorestan University of Medical Sciences, Khorramabad, Iran; ${ }^{6}$ Student Research Committee, Zabol University of Medical Sciences, Zabol, \\ Iran
}

OBJECTIVES: The aim of this study was to assess the quality of life (QoL) of patients with multiple sclerosis (MS), and to investigate the effects of characteristics of MS such as disease course, severity, and relapses on patients' QoL.

METHODS: This was a cross-sectional study, in which 171 patients were enrolled. Health-related QoL was assessed using the Persian version of the Multiple Sclerosis Quality of Life-54 questionnaire. To measure patients' disability status, we used the Expanded Disability Status Scale. Other variables included in the study were disease course and relapses of the disease.

RESULTS: The average scores for patients' physical and mental QoL were $60.9 \pm 22.3$ and $59.5 \pm 21.4$, respectively. In a bivariate analysis, disease course, severity of the disease, and relapses were significantly associated with the physical and mental health composite scores. In a hierarchal regression analysis, disease course, severity of the disease, and relapses were responsible for 38 and $16 \%$ of the variance in physical and mental QoL, respectively. It was also observed that relapses were a strong predictor of both physical and mental QoL.

CONCLUSIONS: Our results showed that disease characteristics significantly affected both dimensions of QoL. It is therefore suggested that health care providers should be aware of these characteristics of MS to more successfully improve MS patients' QoL.

KEY WORDS: Quality of life, Multiple sclerosis, Iran, Disease attributes

\section{INTRODUCTION}

Multiple sclerosis (MS) is one of the most widespread neurological diseases in young adults, and affects approximately 2.3 mil-

\section{Correspondence: Abdollah Almasian Kia}

Department of Health Economics, School of Health Management and Informatin Sciencs, Iran University of Medical Sciences, 6 Rashid Yasemi St, Tehran 1996713883, Iran

E-mail: almasiankia.a@tak.iums.ac.ir

Received: Dec 27, 2016 / Accepted: Feb 19, 2017 / Published: Feb 19, 2017

This article is available from: http://e-epih.org/

(C) This is an open-access article distributed under the terms of the Creative Commons Attribution License (http://creativecommons.org/licenses/by/4.0/), which permits unrestricted use, distribution, and reproduction in any medium, provided the original work is properly cited.

(C) 2017, Korean Society of Epidemiology lion people worldwide. The disease most commonly appears in people aged 20 to 40 years, the age group considered to be the economically active population [1]. MS, after stroke and before Parkinson disease, is considered to be the second most debilitating chronic disease of the central nervous system [2,3]. MS symptoms include impaired coordination and balance, muscle cramps, fatigue, pain, and visual disturbances, and these symptoms can lead to restricted mobility and, in some cases, hospitalization [4,5]. Studies have found that patients suffering from MS experience a quality of life (QoL) lower not only than the general population, but also than those suffering from other chronic diseases [6,7]. Extensive physical disabilities, a lack of effective treatment methods, and the unknown causes of MS provide convincing evidence of the negative impact of this disease on patients' QoL [8]. As MS progresses, patients encounter new manifestations of the disease, with more limitations in their daily activities and working abilities. As 
the disability of patients increases, they become dependent on their family for carrying out their daily routines and activities, which leads to a reduction of their QoL [9].

The concept of QoL in the health field, which is based on patients' individual perspectives, appeared following the development of technologies that increased longevity, and is used as a consideration in the assessment of social policy-making and health care outcomes [10]. Measuring QoL can be a good definition of treatment success, the improvement of a patient's health status after an invasive intervention, and the overall effect of treatment from a patient's perspective [11].

In the field of medicine, researchers often use the concept of health-related quality of life (HRQoL), which specifically assesses the impact of a disease or its treatment on an individual's self-conception of health status and life satisfaction. MS patients' QoL is often measured by the Multiple Sclerosis Quality of Life (MSQoL)-54 questionnaire, which is considered to be the most common and standardized disease-specific instrument for the assessment of QoL of MS patients [12,13].

Improvements can be made in the QoL of MS patients only through understanding effective, behavioral, mental, and social factors [14]. Therefore, studying the QoL of MS patients would help health authorities and policymakers in planning and implementing interventions to increase MS patients' QoL [15]. This study aimed to assess the effect of disease characteristics such as severity, disease course, and relapses in the past three months on the QoL of MS patients.

\section{MATERIALS AND METHODS}

This was a cross-sectional study carried out among MS patients referred to the Center for Special Diseases and Multiple Sclerosis Society of Shiraz between February and October 2013. The inclusion criteria were age greater than 18 years and having at least a 1 -year history of MS. Patients who were not willing to participate in the study or those who had a concomitant chronic disease that would impact their QoL were excluded. Ultimately, 171 patients enrolled in the study and were asked to fill out a questionnaire containing demographic (age, sex, marital status, educational level, and employment status) and clinical (disease duration, relapses during in last three months, and age of onset) questions. Disease course and neurologic disabilities for each patient were measured by a neurologist and recorded by an interviewer. All patients were classified as relapsing-remitting, primary progressive, or secondary progressive based on their disease course. To measure patients' disability status, we used the Expanded Disability Status Scale (EDSS). The Kurtzke EDSS [16] is a quantitative method for assessing MS disability that provides a total score on a scale ranging from 0 , indicating a normal neurological examination, to 10 , indicating death due to MS. Based on their score, patients were categorized in three groups: mild disability (EDSS, 0.0 to 3.5), moderate disability (EDSS, 4.0 to 6.5), and severe disability (EDSS, 7.0 to 9.5).
HRQoL was assessed using the MSQoL-54 questionnaire. This questionnaire is a psychological instrument consisting of 54 standard questions measuring the QoL in MS patients. The MSQoL-54 questionnaire contains 36 questions in a short form for assessing overall health status and general QoL, and 18 questions designed especially for MS patients, with scales for health-related discomfort (4 items); sexual function and satisfaction (5 items); overall QoL (2 items); cognitive function (4 items); and energy, pain, and their social situation ( 3 items). The MSQoL-54 questionnaire assesses health status in two general scales: physical health (39 items) and mental health (15 items). The subscales of physical health include: physical function (assessing possible activities during a normal day), health perceptions, physical role limitations, pain, sexual function, social function, energy, and health distress. The five mental health subscales are as follows: overall QoL, emotional well-being, emotional role limitations (assessing any mental health related problems in working or any other daily activities, such as depression or anxiety), cognitive function (corresponding to problems related to focusing and thinking), and health distress (assessing weight loss and discouragement due to health problems). To calculate the desirability scores for each scale, the weighted average score of the constituent subscales are calculated. These scores range from 0 to 100; in each scale, a higher score means a better QoL.

In this study, we used the Persian version of the MSQoL-54 questionnaire, which was successfully validated by Ghaem \& Haghighi [17], with a Cronbach alpha coefficient of 0.962 for measuring a patient's QoL.

\section{Statistical analysis}

Descriptive statistics such as mean, standard deviation (SD), number and percent were used to analyze all demographic and clinical variables, as well as the physical and mental dimensions of QoL. The Student t-test was used to compare mean differences in the physical and mental health composite scores by sex (male vs. female), marital status (single vs. married), education level (primary vs. secondary or higher), employment status (unemployed vs. employed), disease duration ( $\leq 5$ years vs. $>5$ years), age of onset ( $\leq 30$ years vs. $>30$ years), relapses in the past three months (yes vs. no), disease severity (mild vs. moderate-severe), disease course (relapsing-remitting vs. progressive). The Pearson correlation test was used to assess the relationship between the physical and mental health composite scores and continuous variables, such as disease duration, EDSS scores, and age of onset. To determine the predictors of the physical and mental health composite scores, hierarchical lineal regression was used. We entered two blocks of control variables and one block of predictors in the regression model. The control variables consisted of demographic variables including sex, marital status, education level, and employment status (model 1) and disease duration and age of onset (model 2). The predictors included disease severity, disease course, and relapses in the past three months. The beta coefficient $(\beta), \mathrm{p}$ value, R-squared, adjusted R-squared, and F-value were reported 
for the hierarchical regression models. Data were processed and analyzed using Excel 2007 (Microsoft, Redmond, WA, USA) and Stata version 14.1 (StataCorp., College Station, TX, USA).

\section{Ethics}

This study was approved by the Ethics Committee of the Shiraz University of Medical Sciences and written informed consent was obtained from all patients enrolled in the study.

\section{RESULTS}

Table 1 presents descriptive statistics for the demographic and clinical characteristics of patients. The number (\%) for dichotomous variables and mean (SD) for continuous variables were reported. A total of 171 patients (76.6\% female) participated in this study, with a mean age of 35.7 (8.1) years. Statistically, there was no significant difference between males and females in terms of age $\left(t_{169}=0.24, p=0.8\right.$, t-test). Only $34.0 \%$ of patients were working; the rest were either unemployed or had to quit their job due to MS. The pluralities of participants had a primary education and

Table 1. Demographic and clinical characteristics of the patients

\begin{tabular}{|c|c|c|c|c|}
\hline & Mean \pm SD & n (\%) & Min & Max \\
\hline \multicolumn{5}{|l|}{ Sex } \\
\hline Male & & $40(23.4)$ & 0 & 1 \\
\hline Female & & 131(76.6) & 0 & 1 \\
\hline Age (yr) & $35.7 \pm 8.1$ & & 18 & 61 \\
\hline \multicolumn{5}{|l|}{ Marital status } \\
\hline Single & & $70(41.0)$ & 0 & 1 \\
\hline Married & & $101(59.0)$ & 0 & 1 \\
\hline \multicolumn{5}{|l|}{ Education level } \\
\hline Primary & & $118(69.0)$ & 0 & 1 \\
\hline Secondary or higher & & $53(31.0)$ & 0 & 1 \\
\hline \multicolumn{5}{|l|}{ Employment status } \\
\hline Unemployed & & $113(66.0)$ & 0 & 1 \\
\hline Employed & & $58(34.0)$ & 0 & 1 \\
\hline Disease duration (yr) & $7.6 \pm 4.6$ & & 1 & 24 \\
\hline Age of onset (yr) & $28.1 \pm 7.6$ & & 13 & 50 \\
\hline \multicolumn{5}{|l|}{ Relapses in past 3 months } \\
\hline Yes & & $106(62.0)$ & 0 & 1 \\
\hline No & & $65(38.0)$ & 0 & 1 \\
\hline EDSS score & $2.1 \pm 2.2$ & & 0 & 8.5 \\
\hline \multicolumn{5}{|l|}{ Disease severity } \\
\hline Mild & & $137(80.1)$ & 0 & 1 \\
\hline Moderate & & $20(11.7)$ & 0 & 1 \\
\hline Severe & & $14(8.1)$ & 0 & 1 \\
\hline Disease course & & & 0 & 1 \\
\hline Relapsing-remitting & & $143(83.6)$ & 0 & 1 \\
\hline Secondary progressive & & $22(12.8)$ & 0 & 1 \\
\hline Primary progressive & & $6(3.51)$ & 0 & 1 \\
\hline
\end{tabular}

EDSS, Expanded Disability Status Scale; SD, standard deviation; Min, minimum; Max, maximum. were married. Approximately $83.6 \%$ of patients suffered from relapsing-remitting disease, while the remaining patients were categorized as having primary or secondary progressive disease.

The mean EDSS score was $2.1 \pm 2.2$, and $80.1 \%$ of patients had a mild disability (EDSS, 0.0 to 3.5 ), while 11.7 and $8.1 \%$ were categorized as having a moderate disability (EDSS, 4.0 to 6.5) and a severe disability (EDSS, 7.0 to 9.5), respectively. The distribution of patients by EDSS score is presented in Figure 1.

The average scores for patients' physical and mental QoL were $60.9 \pm 22.3$ and 59.5 \pm 21.4 , respectively. According to the QoL subscale scores, social function and energy had the highest scores $(74.1 \pm 24.6)$ and the lowest scores $(51.8 \pm 21.2)$, respectively. Figure 2 presents the mean scores of the physical and mental subscales in our study.

Table 2 illustrates the relationships of the physical and mental dimensions of QoL with the demographic and clinical characteristics of patients. Females reported higher scores in the physical

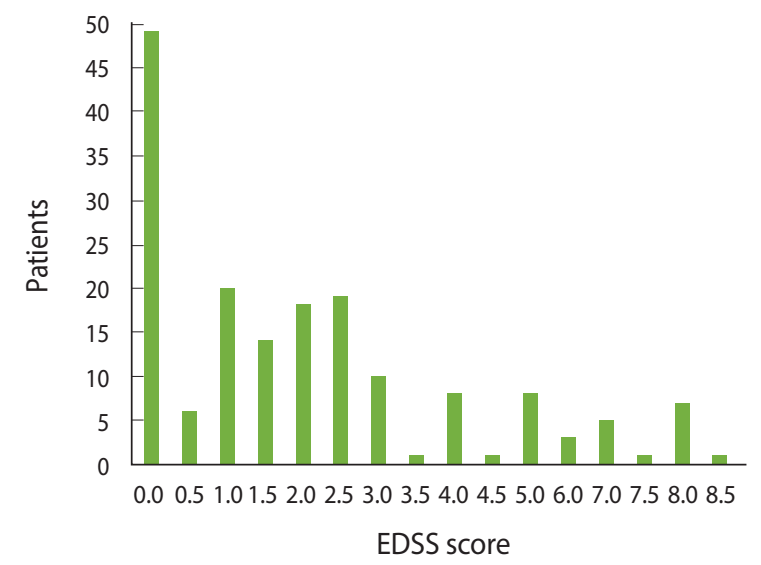

Figure 1. Distribution of patients by Expanded Disability Status Scale (EDSS) score.

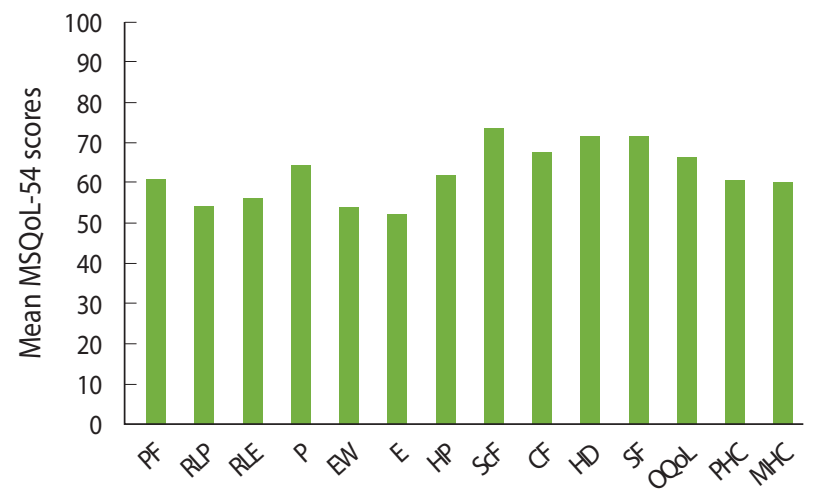

Figure 2. Mean scores of the Multiple Sclerosis Quality of Life (MSQoL)54 subscales. PF, physical function; RLP, role limitation-physical; RLE, role limitation-emotional; $P$, pain; EW, emotional well-being; $E$, energy; $\mathrm{HP}$, health perception; ScF, social function; $\mathrm{CF}$, cognitive function; $H D$, health distress; SF, sexual function; OQoL, overall quality of life; $\mathrm{PHC}$, physical health composite; MHC, mental health composite. 
Table 2. Mean physical and mental health composite scores by demographic and clinical variables

\begin{tabular}{|c|c|c|}
\hline & Physical health & Mental health \\
\hline \multicolumn{3}{|l|}{ Sex } \\
\hline Male & $54.37 \pm 3.89^{*}$ & $56.73 \pm 3.53$ \\
\hline Female & $62.90 \pm 1.86$ & $60.40 \pm 1.84$ \\
\hline \multicolumn{3}{|l|}{ Marital status } \\
\hline Single & $62.10 \pm 2.17$ & $59.12 \pm 2.09$ \\
\hline Married & $59.18 \pm 2.76$ & $60.15 \pm 2.63$ \\
\hline \multicolumn{3}{|l|}{ Education level } \\
\hline Primary & $59.61 \pm 2.09$ & $58.52 \pm 1.95$ \\
\hline Secondary or higher & $63.94 \pm 3.00$ & $61.87 \pm 3.05$ \\
\hline \multicolumn{3}{|l|}{ Employment status } \\
\hline Unemployed & $62.68 \pm 2.02$ & $60.09 \pm 1.98$ \\
\hline Employed & $57.71 \pm 3.08$ & $58.55 \pm 2.89$ \\
\hline \multicolumn{3}{|l|}{ Disease duration (yr) } \\
\hline$\leq 5$ & $66.01 \pm 2.72^{* *}$ & $60.53 \pm 2.91$ \\
\hline$>5$ & $57.70 \pm 2.14$ & $58.91 \pm 1.94$ \\
\hline \multicolumn{3}{|l|}{ Age of onset (yr) } \\
\hline$\leq 30$ & $63.52 \pm 2.17^{*}$ & $61.94 \pm 2.11^{*}$ \\
\hline$>30$ & $56.21 \pm 2.69$ & $55.20 \pm 2.50$ \\
\hline \multicolumn{3}{|c|}{ Relapses in past 3 months } \\
\hline Yes & $53.85 \pm 2.07^{* * *}$ & $54.21 \pm 2.07^{* * *}$ \\
\hline No & $72.41 \pm 2.36$ & $68.22 \pm 2.30$ \\
\hline \multicolumn{3}{|l|}{ Disease severity } \\
\hline Mild & $66.42 \pm 1.75^{* * *}$ & $62.18 \pm 1.79 * * *$ \\
\hline Moderate-severe & $38.68 \pm 2.48$ & $48.89 \pm 3.44$ \\
\hline \multicolumn{3}{|l|}{ Disease course } \\
\hline Relapsing-remitting & $65.10 \pm 1.73^{* * *}$ & $61.75 \pm 1.74^{* * *}$ \\
\hline Progressive & $39.48 \pm 3.40$ & $48.24 \pm 4.01$ \\
\hline
\end{tabular}

Values are presented as mean \pm standard deviation.

${ }^{*} p<0.05,{ }^{* *} p<0.01,{ }^{* * *} p<0.001$ by the Student t-test.

dimension than males $(\mathrm{p}<0.05)$, while there was no significant difference in the mean mental composite score between males and females. Both physical and mental scores did not show statistically significant differences according to marital status, education level, or employment status.

Individuals suffering from relapsing-remitting disease had a significantly higher level of QoL in both the physical and mental dimensions. The relationship between QoL and disease relapse in the past three months was significant, such that patients who had experienced relapse had lower levels of physical and mental QoL than those who had not. Additionally, patients with an EDSS > 3.5, a disease duration of $>5$ years, and an age of onset of $>30$ years had the poorest QoL. These findings were also statistically significant.

Table 3 indicates that linear relationships were present between the dimensions of QoL and EDSS scores, disease duration, and age of onset. Based on the Pearson correlation test, EDSS scores showed a strong negative correlation with the physical health composite scores and a moderate negative correlation with the mental
Table 3. Correlations between disease duration, age of onset, EDSS score, and health-related quality of life

\begin{tabular}{llcc}
\hline Composite & $\begin{array}{c}\text { Disease } \\
\text { duration }\end{array}$ & $\begin{array}{c}\text { Age of } \\
\text { onset }\end{array}$ & $\begin{array}{c}\text { EDSS } \\
\text { score }\end{array}$ \\
\hline Physical health & $-0.259^{* * *}$ & -0.128 & $-0.693^{* * *}$ \\
Mental health & -0.085 & -0.140 & $-0.428^{* * *}$ \\
\hline
\end{tabular}

EDSS, Expanded Disability Status Scale.

*** $p<0.001$ by pair-wise Pearson correlations.

health composite scores. We observed a weak negative relationship between the physical dimension of QoL and disease duration, whereas the mental score did not show any association with the duration of disease. The correlation between age of onset and both composite QoL scores did not have statistical significance.

Hierarchical regression was used to assess the effects of demographic and clinical characteristics on the physical and mental health composite scores. As shown in Table 4, the total variance of the physical and mental dimensions that was explained by all variables was 0.382 and 0.166 , respectively. After controlling for demographic variables (model 1), other variables, such as disease duration, age of onset, disease course, disease severity, and relapses in the past three months, were responsible for $35 \%$ of the variance in the physical composite score and $15 \%$ of the variance in the mental composite score. Disease severity and relapses in the past three months were the most significant determinants of physical status $(\mathrm{p}<0.001)$, and relapses were the most significant predictor variable for the mental dimension.

\section{DISCUSSION}

The aim of this study was to assess the effects of MS characteristics such as disease severity, disease course, and relapses in the past three months on the physical and mental dimensions of QoL in patients with MS. Our findings highlight that disease characteristics were especially important factors for the physical health composite score, more so than for the mental health composite score. Demographic and clinical characteristics explained 38\% of the variance in the physical health dimension and $16 \%$ of the variance in the mental health composite score.

According to our results, there were no significant relationships between demographic factors and QoL in MS patients, except for sex and the physical health composite score. Some other studies conducted in Iran also reported that demographic characteristics were unrelated to HRQoL in MS patients [18-21]. In contrast, some studies showed that being female [22], having a lower education level [23], and being older [24] were factors related to poorer QoL.

In this study, we found a statistically significant negative relationship between the duration of disease and the physical dimension of QoL $(r=-0.25, \mathrm{p}<0.001)$ which shows that a longer duration of the disease was associated with a poorer QoL. A negative relationship, but not a significant one, was observed between the duration of the disease and the mental dimension of QoL $(r=-0.08$, $\mathrm{p}=0.25$ ). Some studies have also stated that the duration of the 
Table 4. Results of hierarchical regression assessing the effects of demographic and clinical variables on physical- and mental health composite scores

\begin{tabular}{|c|c|c|c|c|c|c|}
\hline \multirow{3}{*}{ Variable } & \multicolumn{3}{|c|}{ Physical health } & \multicolumn{3}{|c|}{ Mental health } \\
\hline & \multicolumn{3}{|c|}{ Coefficients } & \multicolumn{3}{|c|}{ Coefficients } \\
\hline & Model 1 & Model 2 & Model 3 & Model 1 & Model 2 & Model 3 \\
\hline \multicolumn{7}{|l|}{ Sex } \\
\hline Female & 6.196 & 6.876 & 3.334 & 3.296 & 3.873 & 0.613 \\
\hline \multicolumn{7}{|l|}{ Marital status } \\
\hline Single & 2.744 & $9.526^{*}$ & 6.322 & -0.970 & 3.142 & 1.277 \\
\hline \multicolumn{7}{|l|}{ Employment status } \\
\hline Employed & -1.830 & 0.915 & 3.210 & -0.370 & 1.514 & 2.607 \\
\hline \multicolumn{7}{|l|}{ Education level } \\
\hline Secondary or higher & 4.814 & 2.792 & -0.002 & 2.857 & 1.597 & -0.206 \\
\hline Disease duration (yr) & & $-1.436^{* * *}$ & -0.416 & & -0.473 & 0.124 \\
\hline Age of onset (yr) & & $-0.668^{* *}$ & $-0.470^{*}$ & & -0.486 & -0.415 \\
\hline \multicolumn{7}{|l|}{ Disease course } \\
\hline Progressive & & & -4.483 & & & -5.066 \\
\hline \multicolumn{7}{|l|}{ Disease severity } \\
\hline Moderate-severe & & & $-15.085^{* *}$ & & & -3.101 \\
\hline \multicolumn{7}{|c|}{ Relapses in past 3 months } \\
\hline None & & & $14.734^{* * *}$ & & & $13.038^{* * *}$ \\
\hline Constant & $53.750^{* * *}$ & $78.611^{* * *}$ & $73.690^{* * *}$ & $56.866^{* * *}$ & $70.982^{* * *}$ & $60.732^{* * *}$ \\
\hline $\mathrm{n}$ & 170 & 170 & 170 & 170 & 170 & 170 \\
\hline $\mathrm{F}$ & 1.55 & $4.58^{* * *}$ & $11.02^{* * *}$ & 0.42 & 1.08 & $3.55^{* * *}$ \\
\hline R-squared & 0.036 & 0.144 & 0.382 & 0.010 & 0.038 & 0.166 \\
\hline Adjusted R-squared & 0.012 & 0.112 & 0.348 & -0.013 & 0.002 & 0.119 \\
\hline
\end{tabular}

${ }^{*} \mathrm{p}<0.05,{ }^{* *} \mathrm{p}<0.01,{ }^{* * *} \mathrm{p}<0.001$ by hierarchical linear regression.

disease negatively affected patients' QoL [25-29]. However, other studies have reported contradictory results, with some indicating that there was no relationship between the duration of disease and QoL $[24,30]$. It seems that along duration of MS, with corresponding physical limitations, leads to a decrease in QoL in the physical dimension.

In the present study, patients with relapsing-remitting MS had better physical and mental QoL than patients with progressive MS. Mitchell et al. [15] showed that the disease course negatively affected patients' QoL. The more aggressive the disease course was, the poorer the QoL. Tadić et al. [25] reported that since the disease develops faster in MS patients with a progressive disease course, their QoL is negatively affected.

The results of our study showed a negative relationship between the QoL score and severity of the disease, such that patients with less severe disease had a better QoL. In previous studies, a strong negative correlation between disability status and QoL has been reported [2,31-33]. Regression analysis showed that the severity of the disease was the most relevant independent variable predicting variance in the physical dimension of patients' QoL. Szilasiova et al. [29] reported that disability was significantly related to both the physical and mental dimensions of QoL; but after adjusting the regression model to account for depression and anxiety, no significant relationship was observed between disability and the mental dimension of QoL. The contribution of disability level of MS to QoL variation has been reported to range from $2 \%$ in the US to $29 \%$ in Austria. This wide variation is due to the use of different questionnaires for assessing QoL [34]. Petersen et al. [35] argued that physicians mostly pay attention to the physical dimensions of a disease, while from the patients' point of view; mental health is an important QoL determinant. Therefore, it should be noted that the EDSS is not able to provide information about the mental health dimension.

In the present study, it was also observed that relapse of the disease was a strong predictor of both physical and mental QoL. A study conducted by Miller et al. [36] showed that more relapses in the last two years were associated with poorer QoL. They also found that preventing disease relapse led to improvements in QoL. Jones et al. [37] also found that patients who experienced relapses had poorer HRQoL than those with no relapses. In another study, Mäurer et al. [38] showed that patients who had experienced severe relapses had significantly poorer HRQoL. Healy et al. [39] reported a relationship between relapses and HRQoL, in which patients with more severe relapses had a greater decline in HRQoL than those with mild relapses.

Our study has some limitations that should be considered. 
First, caution should be used in interpreting our findings as indicative of a causal relationship between QoL and disease characteristics because of the cross-sectional study design. Second, the sample size of participant was somewhat small. Third, we did not consider variables such as fatigue, depression, anxiety, and social support, which might also affect QoL.

Briefly, our study showed that both the physical and mental dimensions of MS patients' QoL were significantly affected by disease characteristics, such as its severity and the occurrence of relapses. It is therefore suggested that health care providers should be appropriately informed about these characteristics of MS, in order to more successfully improve MS patients' QoL.

\section{CONFLICT OF INTEREST}

The authors have no conflicts of interest to declare for this study.

\section{ORCID}

Aziz Rezapour: http://orcid.org/0000-0002-5583-5561; Abdollah Almasian Kia: http://orcid.org/0000-0001-6944-9023; Sahar Goodarzi: $h t t p: / / o r c i d . o r g / 0000-0002-6389-3593 ;$ Mojtaba Hasoumi: $h t t p$ : //orcid.org/0000-0002-0900-1590; Soraya Nouraei Motlagh: http:// orcid.org/0000-0003-4121-2205; Sajad Vahedi: http://orcid.org/00000002-5698-0699

\section{REFERENCES}

1. Browne P, Chandraratna D, Angood C, Tremlett H, Baker C, Taylor BV, et al. Atlas of Multiple Sclerosis 2013: a growing global problem with widespread inequity. Neurology 2014;83:1022-1024.

2. Kargarfard M, Eetemadifar M, Mehrabi M, Maghzi AH, Hayatbakhsh MR. Fatigue, depression, and health-related quality of life in patients with multiple sclerosis in Isfahan, Iran. Eur J Neurol 2012;19:431-437.

3. Opara J, Jaracz K, Brola W. Burden and quality of life in caregivers of persons with multiple sclerosis. Neurol Neurochir Pol 2012; 46:472-479.

4. Basić Kes V, Čengić L, Cesarik M, Tomas AJ, Zavoreo I, Matovina LZ, et al. Quality of life in patients with multiple sclerosis. Acta Clin Croat 2013;52:107-111.

5. Tan H, Yu J, Tabby D, Devries A, Singer J. Clinical and economic impact of a specialty care management program among patients with multiple sclerosis: a cohort study. Mult Scler 2010;16:956-963.

6. Lerdal A, Celius EG, Moum T. Perceptions of illness and its development in patients with multiple sclerosis: a prospective cohort study. J Adv Nurs 2009;65:184-192.

7. Fernández-Muñoz JJ, Morón-Verdasco A, Cigarán-Méndez M, Muñoz-Hellín E, Pérez-de-Heredia-Torres M, Fernández-de-lasPeñas C. Disability, quality of life, personality, cognitive and psychological variables associated with fatigue in patients with multiple sclerosis. Acta Neurol Scand 2015;132:118-124.

8. Akhani A, Habibi M, Izadikhah Z, Monajemi MB, Jamshinejad N.
Quality of life model in multiple sclerosis: personality, mood disturbance, catastrophizing and disease severity. Int J Indian Psychol 2015;3:C00227V3I12015.

9. Forbes A, While A, Mathes L. Informal carer activities, carer burden and health status in multiple sclerosis. Clin Rehabil 2007;21: 563-575.

10. Plummer M, Molzahn AE. Quality of life in contemporary nursing theory: a concept analysis. Nurs Sci Q 2009;22:134-140.

11. Guimarães FA, Oliveira-Cardoso EA, Mastropietro AP, Voltarelli JC, Santos MA. Impact of autologous hematopoetic stem cell transplantation on the quality of life of patients with multiple sclerosis. Arq Neuropsiquiatr 2010;68:522-527.

12. Bandari DS, Vollmer TL, Khatri BO, Tyry T. Assessing quality of life in patients with multiple sclerosis. Int J MS Care 2010;12:3441.

13. Opara JA, Jaracz K, Brola W. Quality of life in multiple sclerosis. J Med Life 2010;3:352-358.

14. Motl RW, McAuley E, Snook EM, Gliottoni RC. Physical activity and quality of life in multiple sclerosis: intermediary roles of disability, fatigue, mood, pain, self-efficacy and social support. Psychol Health Med 2009;14:111-124.

15. Mitchell AJ, Benito-León J, González JM, Rivera-Navarro J. Quality of life and its assessment in multiple sclerosis: integrating physical and psychological components of wellbeing. Lancet Neurol 2005;4:556-566.

16. Kurtzke JF. Rating neurologic impairment in multiple sclerosis: an expanded disability status scale (EDSS). Neurology 1983;33: 1444-1452.

17. Ghaem H, Borhani Haghighi A. The impact of disability, fatigue and sleep quality on the quality of life in multiple sclerosis. Ann Indian Acad Neurol 2008;11:236-241.

18. Salehi R, Shakhi K, Khiavi FF. Association between disability and quality of life in multiple sclerosis patients in Ahvaz, Iran. Mater Sociomed 2016;28:215-219.

19. Ashjazadeh N, Hadianfard H, Feridoni S, Farjam E. Assessment of health-related quality of life in patients with multiple sclerosis living in the Fars province of Iran. Neuroimmunol Neuroinflamm 2016;3:57-62.

20. Abolfazli R, Hosseini A, Gholami Kh, Javadi MR, Torkamandi H, Emami S. Quality of life assessment in patients with multiple sclerosis receiving interferon beta-1a: a comparative longitudinal study of Avonex and its biosimilar CinnoVex. ISRN Neurol 2012;2012: 786526.

21. Ayatollahi P, Nafissi S, Eshraghian MR, Kaviani H, Tarazi A. Impact of depression and disability on quality of life in Iranian patients with multiple sclerosis. Mult Scler 2007;13:275-277.

22. Sahebalzamani M, Zamiri M, Rashvand F. The effects of self-care training on quality of life in patients with multiple sclerosis. Iran J Nurs Midwifery Res 2012;17:7-11.

23. Milani MJ, Ashktorab T, Saeedi JA, Majd HA. The effect of illness perception on physical health-related quality of life promotion in multiple sclerosis (MS) patients attending peer support groups. J Paramed Sci 2012;3:31-37. 
24. Salehpoor G, Hosseininezhad M, Rezaei S. A preliminary path analysis: effect of psychopathological symptoms, mental and physical dysfunctions related to quality of life and body mass index on fatigue severity of Iranian patients with multiple sclerosis. Iran J Neurol 2012;11:96-105.

25. Tadić D, Dajić V. Quality of life in patients with multiple sclerosis in Republic of Srpska. Med Glas (Zenica) 2013;10:113-119.

26. Kehler MD. Emotional adjustment to multiple sclerosis: evaluation of a stress and coping model and a cognitive adaptation model [dissertation]. Regina: University of Regina; 2013.

27. Janardhan V, Bakshi R. Quality of life in patients with multiple sclerosis: the impact of fatigue and depression. J Neurol Sci 2002; 205:51-58.

28. Patti F, Cacopardo M, Palermo F, Ciancio MR, Lopes R, Restivo D, et al. Health-related quality of life and depression in an Italian sample of multiple sclerosis patients. J Neurol Sci 2003;211:55-62.

29. Szilasiova J, Krokavcova M, Gdovinova Z, Rosenberger J, Van Dijk JP. Quality of life in patients with multiple sclerosis in Eastern Slovakia. Disabil Rehabil 2011;33:1587-1593.

30. Cecilie Fjeldstad C, Pardo G, Bemben M. Quality of life according to duration of disease in women with low disability in multiple sclerosis. Int J MS Care 2008;10:77-80.

31. Göksel Karatepe A, Kaya T, Günaydn R, Demirhan A, Ce P, Gedizlioğlu M. Quality of life in patients with multiple sclerosis: the impact of depression, fatigue, and disability. Int J Rehabil Res 2011; 34:290-298.

32. Baumstarck K, Pelletier J, Boucekine M, Auquier P; MusiQoL Study Group. Predictors of quality of life in patients with relapsing-remitting multiple sclerosis: a 2-year longitudinal study. Rev Neurol (Paris) 2015;171:173-180 (French).
33. Twork S, Wiesmeth S, Spindler M, Wirtz M, Schipper S, Pöhlau D, et al. Disability status and quality of life in multiple sclerosis: nonlinearity of the Expanded Disability Status Scale (EDSS). Health Qual Life Outcomes 2010;8:55.

34. Papuć E, Stelmasiak Z. Factors predicting quality of life in a group of Polish subjects with multiple sclerosis: accounting for functional state, socio-demographic and clinical factors. Clin Neurol Neurosurg 2012;114:341-346.

35. Petersen JS, Kyvik KO, Bingley PJ, Gale EA, Green A, Dyrberg T, et al. Population based study of prevalence of islet cell autoantibodies in monozygotic and dizygotic Danish twin pairs with insulin dependent diabetes mellitus. BMJ 1997;314:1575-1579.

36. Miller DM, Weinstock-Guttman B, Bourdette D, You X, Foulds P, Rudick RA. Change in quality of life in patients with relapsingremitting multiple sclerosis over 2 years in relation to other clinical parameters: results from a trial of intramuscular interferon \{beta\}-1a. Mult Scler 2011;17:734-742.

37. Jones E, Pike J, Marshall T, Ye X. Quantifying the relationship between increased disability and health care resource utilization, quality of life, work productivity, health care costs in patients with multiple sclerosis in the US. BMC Health Serv Res 2016;16:294.

38. Mäurer M, Comi G, Freedman MS, Kappos L, Olsson TP, Wolinsky JS, et al. Multiple sclerosis relapses are associated with increased fatigue and reduced health-related quality of life: a post hoc analysis of the TEMSO and TOWER studies. Mult Scler Relat Disord 2016;7:33-40.

39. Healy BC, Degano IR, Schreck A, Rintell D, Weiner H, Chitnis T, et al. The impact of a recent relapse on patient-reported outcomes in subjects with multiple sclerosis. Qual Life Res 2012;21:16771684. 